\title{
An Approach for Object Detection in Multi Temporal Aerial Images
}

\author{
Tapasmini Sahoo \\ Department of Electronics and \\ Communication Engineering \\ ITER, Siksha 'O' Anusandhan University \\ Bhubaneswar, India
}

\author{
Kunal Kumar Das \\ Department of Electronics and \\ Communication Engineering \\ ITER, Siksha 'O' Anusandhan University \\ Bhubaneswar, India
}

\begin{abstract}
In this paper an image fusion technique is developed to detect manmade structures from multi-temporal images. Proposed method is a hybrid approach. Histogram based spectral analysis is used to remove contamination of clouds and their shadows recursively that integrates complimentary information to form a composite image from multi temporal images. Then the algorithm tries to extract edge information using discrete wavelet approach. In this work a recursive threshold based segmentation approach is used for removal of clouds and their shadows but wavelet transform is adopted for the image fusion to reduce artifacts in the fused image. Further a feature-based fusion rule is used to reduce the computing time. The proposed method is used for building detection and results show that the proposed method performs well.
\end{abstract}

\section{Keywords}

Histogram, Wavelet Transform, Recursive

\section{INTRODUCTION}

A significant obstacle of extracting information using remote sensing imagery is the presence of clouds and their shadows. Removing these portion of an image and then filling in the missing data ia an important image editing task. Traditionally the objective is to cut the cloudy portions out from the frame and fill in the gaps with clear patches from similar images taken at a different time. Thus the meticulous work is to identify the cloud and then completing the missing parts. Various approaches have been tried to solve this problem with differing results. Wavelet transformation has showed to be a useful tool to deal with clouds and shadows coverage in remote sensing images. In this paper the performance of stationary wavelet transformation with multi scale product approach for detection of clouds from SAR image is studied and a histogram based threshold approach is proposed to remove clouds without any wavelet synthesis and analysis. The scheme performs better than the simple pattern recognition method and as well as the wavelet transforms methods of detection. The performance of the proposed scheme is demonstrated experimentally for various SAR images.

Satellite image acquisition systems are generating more data than can be analyzed by human experts. In the second part of this paper an automated detection and recognition of buildings in presence of cloud in satellite imageries has been explored. Local changes or variations of the intensity of imagery (such as edges \& corners) are important information for image processing and pattern recognition. Wavelet analysis is one of the most popular techniques that can be used to detect local intensity variation [13]. This technique is coupled with the canny edge detection, which has the double threshold technique $\&$ is less fooled by noise, forms a very strong tool in the detection of manmade features such as buildings. So applying stationary wavelet based image fusion a cloud free SAR image of building is found \& then applying the edge extraction technique the edges of building is detected.

\section{WAVELET APPROACH FOR REMOVAL OF CLOUD AND CLOUD SHADOWS}

Wang [9] proposed automated cloud detection in a set of two temporal Land sat TM images by simply thresholding high frequency components as extracted by a $2 \mathrm{D}$ discrete wavelet transform of both images. That study suggests an automated use of Discrete Wavelet Frames (DWF) in order to detect clouds and their shadows in satellite images. The special frame mentioned in his paper can be called undecimated wavelet transform or stationary wavelet transform (SWT).

\subsection{Decimated wavelet approach}

Compared with the orthogonal wavelet transform with Mallat algorithm, the wavelet coefficients from the wavelet frame are translation-invariant. For the image texture, you may acquire the same image with a sample shift. If you apply the traditional wavelet transform, the wavelet coefficients will change. But for the wavelet frame, there is only 1 sample shift in the coefficients. Such property is very suitable in signal/image feature extraction although the redundancy from the frame is harmful for signal/image compression. This property is very useful for several applications such as breakdown point's detection.

The drawback of the DWT is that it is not a time-invariant transform. This means that, even with periodical signal extension, the DWT of a translated version of a signal X is not, in general, the translated version of the DWT of X. The idea is to average some slightly different DWT, called "decimated DWT, to define the stationary wavelet transform (SWT). The SWT is similar to a discrete wavelet transformation (DWT). The difference between both of them is that SWT does not perform down sampling of the signal between the levels of hierarchy. For that reason the sub images obtained after the decomposition process have the same resolution as the original image.

\subsection{Multiscale product using undecimated wavelet transform}

The previous approach has been focused on detecting clouds and their shadows only one decomposition level. This approach performs a detection of clouds and their shadows using two wavelet decomposition levels. Carvalho [3] mentioned that clouds and their shadows appear as peaks of narrow bandwidth in the temporal spectrum (time series). In the spatial domain they appear in a similar way but with 
variable bandwidth. If we consider the presence of clouds and their shadows as signal response against a "noisy" background, a framework for their detection can be set forth based on noise modeling in transformed space.

Figure 1 shows the multi-scale product process using SWT. First of all wavelet decomposition is achieved at two decomposition levels. Details images are added for each decomposition level. As a result we obtained two added images, one for each decomposition level. After that, multiscale product is joined with the previous decomposed images. Multi-scale point-wise product was applied in order to further enhance singularities (i.e., edges and peaks) in the signal. That inter-scale correlation is represented by equation 1 .

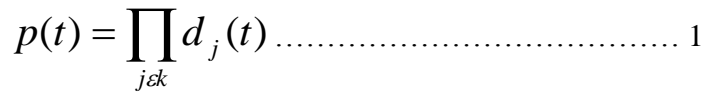

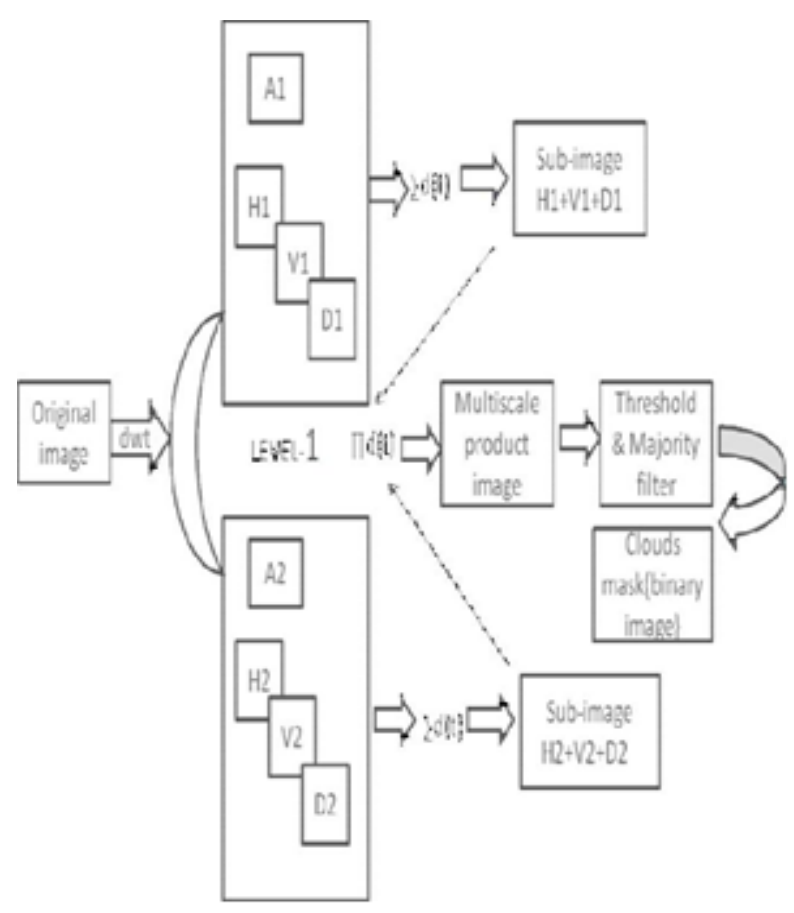

Fig 1: Multi-scale product approach

\section{DETECTION OF CLOUD USING HISTOGRAM BASED THRESHOLD APPROACH}

This approach to detect clouds and their shadows allows us to compare with the two previous wavelet approaches in order to know the accuracy of each methodology. The procedure is showed in Figure 2. This approach takes into account at least two satellite images of the same area at different times. As a pre-processing condition, those images have to be coregistered. That process ensures that the same pixel in both images refers to the same area. Because images from the same area but different time have different solar irradiation and atmospheric effects, it is necessary to reduce those effects. It is done through a reflectance correction process that was applied for this approach.

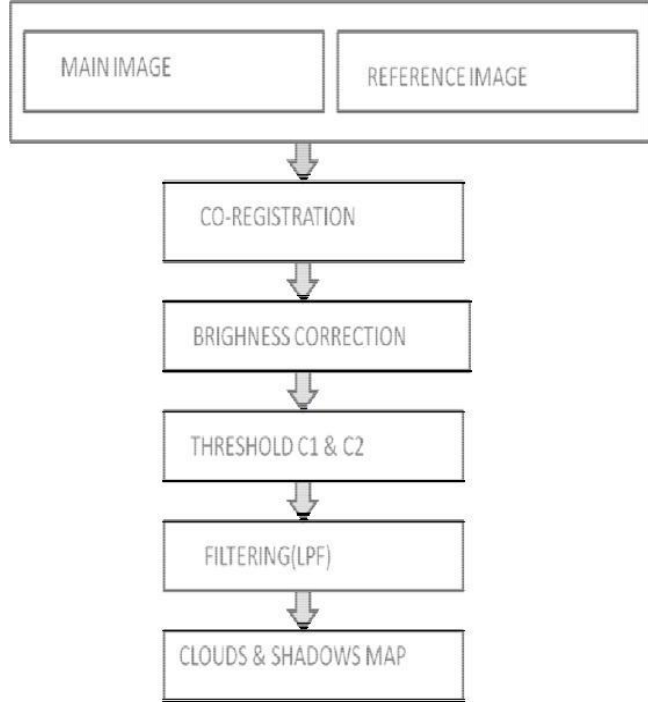

Fig 2: Histogram based threshold approach

\subsection{Reflectance Correction}

A linear relation was considered in order to reduce the different solar irradiance and atmospheric effects on the brightness value of the two or more images. The main image is considered as standard and the reference image is adjusted.

$$
I_{r e f}^{\prime}(i, j)=\frac{\sigma_{\text {main }}}{\sigma_{\text {ref }}}\left[I_{r e f}(i, j)-\mu_{\text {ref }}\right]+\mu_{\text {main }} \ldots 2
$$

Where Iref $(i, j)$ is the previous brightness(reflectance) value of a pixel of the reference image. $\mu$ ref and $\mu$ main are the mean of the reference and main images respectively. $\sigma$ ref and $\sigma$ main are the standard deviation of the reference image and the standard deviation of the main image respectively.

\subsection{Detection of clouds and their shadows}

Using the two previously corrected images, we set a threshold $\mathrm{T} 1$ to differentiate the clouded regions and the shadowed regions in the images. It is important to take into account that clouds reflect the solar radiation higher than the ground in the visible and infrared bands. On the contrary, shadow areas do not get directly solar radiation, for that reason we always obtain very low DN values. Consider only the previous threshold is not a guarantee for a correct detection of clouds and shadows. Many features on the ground have similar DN value as clouds (i.e., building areas, greenhouses, bare soil) and also other features show a reflection very similar to that of shadows (i.e., water bodies, topographic shadows). But that object does not have a unique property that clouds and shadows have. That is movement. This is way, in addition to the previous threshold $\mathrm{c} 1$ we need to define another threshold, this time $\mathrm{c} 2$.

To be sure about a correct detection, the absolute brightness difference between the same locations of the main image and the reflectance-corrected reference image is compared with threshold T2. Equation 3 shows this procedure for clouds and shadows detection.

$((\operatorname{Imain}(, j)>T 1) \mathrm{U}(\mathrm{I} \operatorname{ref}(i, j)>T 1)) \mathrm{I}((\operatorname{Imain}(, j)-\mathrm{I} \operatorname{ref}(i$,

j)) $>T 2$ ) 


\section{IMAGE FUSION}

There are many approaches of image fusion. Wavelet Domain of image fusion is one of them. Wavelets have been used to fuse high-resolution panchromatic images and low resolution multi spectral images. Those approaches have shown better results than more traditional methods. Also, fusion process for images of the same spatial and spectral characteristics has been done in previous studies. That information corresponds to different dates (multi temporal data). Different wavelet transformation methods have been applied. Image fusion have been produced using Wavelet Packages (WP), Discrete wavelet frames (DWF) [11], [9], [12] and multi- band transformation. Non-separable wavelet frame (NWF) performed better than discrete wavelet frame (DWF). All of them perform better than the discrete wavelet transformation (DWT).

\subsection{Undecimated wavelet based image fusion}

In this paper in order to fill out the missing information caused by clouds and their shadows in the satellite images, image fusion using wavelet approach is applied, as shown in Figure 3. For this process, two co- registered images are selected. In this case, a cloud-free image was selected as main image and a clouded image as reference image. Preprocessing stage correction of the two selected images was applied following the procedure explained in Equation 3.

Undecimated (Stationary) wavelet transformation at level one was applied to both images. The resulting sub- images have the same size as the original images. Results detection of clouds and their shadows have been recorded on the binary decision maps. By using the two maps, the undecimated wavelets transform values of the detailed information for each pixel was integrated. As a result a fused image is constructed by performing an inverse wavelet transformation (IWT) based on the combined transform values.

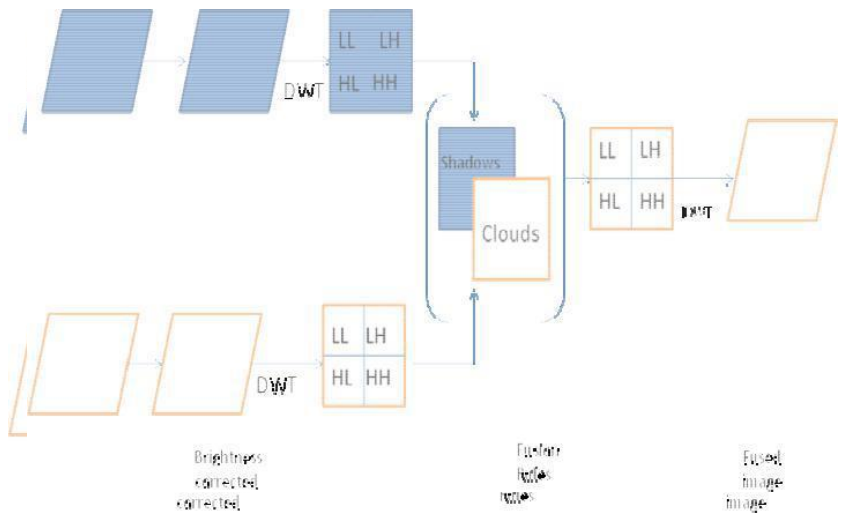

Fig 3: Image fusion technique

The wavelet transform provides both spatial and frequency domain localization. The two binary decision maps are used for controlling purpose so that the required information corresponding to the cloud and shadow regions of the reference image is extracted from the main image and integrated into the reference image. Thus a cloud free fused image was obtained.

\subsection{Edge Detection}

Edges of an image reflect the information of the image. They contain the basic character of the image. Edges within an image correspond to intensity discontinuities that result from different surface reflectance of objects, various illumination conditions, or varying distance and orientations of objects from a viewer. Edge detection is a common problem and of fundamental importance in image analysis and computer vision. Edges however generally occur at various resolutions, or scales, and represent transitions of different degrees, or gradient levels. Perhaps the most commonly used method for detecting edges in an image is through the spatial gradient. In this approach, the edges are identified by the local extrema in the differentiated image through thresholding. The Canny edge detector has been used considering various factors; one important feature being that this edge detector is less likely to be "fooled" by noise and more likely to detect true weak edges, which are very important for the detection of building edges. The double thresholding of the Canny edge detector plays the main role in edge detection. The method uses two thresholds to detect strong edges and weak edges, and includes the weak edges in the output only if they are connected to strong edges. The Canny operator works in a multi-stage process. The Canny edge detection algorithm first smoothens the image with a Gaussian filter and then computes the gradient magnitude and orientation using finite-difference approximations for the partial derivatives and applies nonmaxima suppression to the gradient magnitude. Eventually using the double threshold algorithm detects and links the edges.

The upper tracking threshold can be set to quite high and lower threshold quite low for good results. Setting the lower threshold too high will cause noisy edges to break up. Setting the upper threshold too low increases the number of spurious and undesirable edge fragments appearing in the output.

\subsection{Proposed method of edge extraction of buildings in SAR images}

The block diagram for edge extraction of buildings in SAR images is shown in the figure. 4 shown below. The extracted edge map by mentioned method is cleaned by some morphological operators. As per this method at the initial stage wavelet transform is applied to decompose the input image and then is passed through canny edge detector. After edge extraction from the SAR image we can also extract different features from the image by applying simple image segmentation technique.

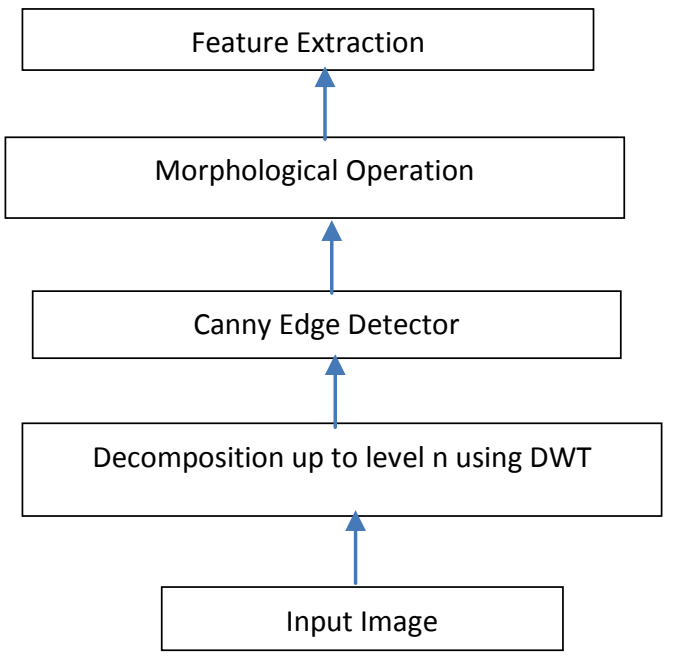

Fig 4: Edge Detection Technique

\section{RESULT AND DISCUSSION}

In this paper we applied the proposed method to detect and remove cloud from the SAR image and also detected the edges of building. The SAR image corresponds to the 
SimCity-small area of America. Several images are acquired and have been registered using image to image registration process.

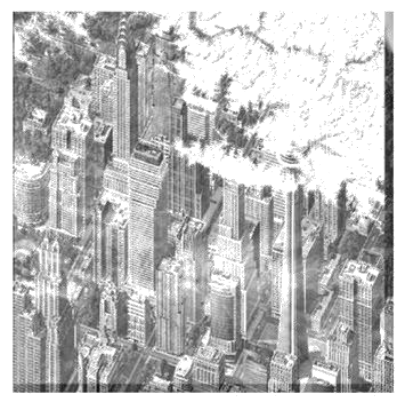

$a$

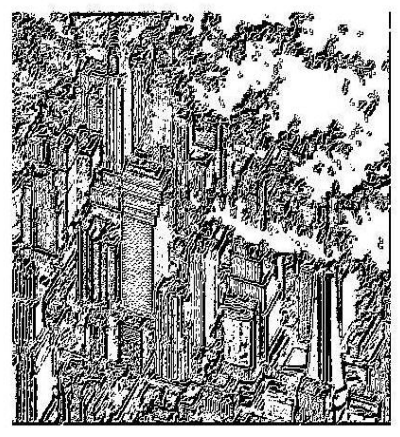

$b$

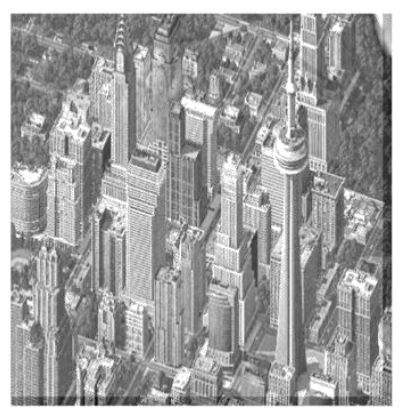

$c$

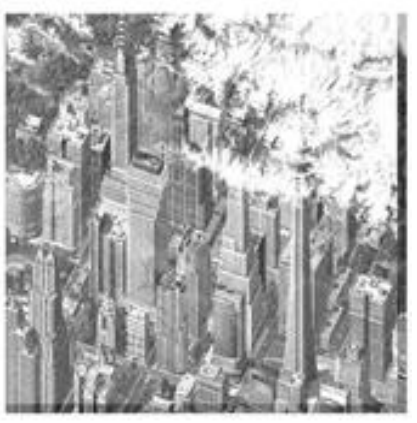

$d$

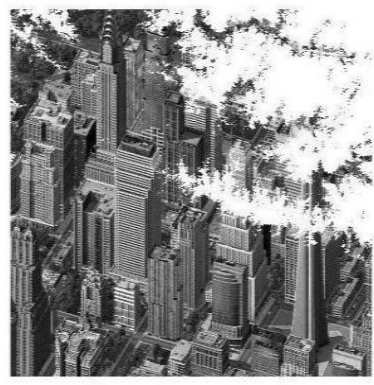

$e$

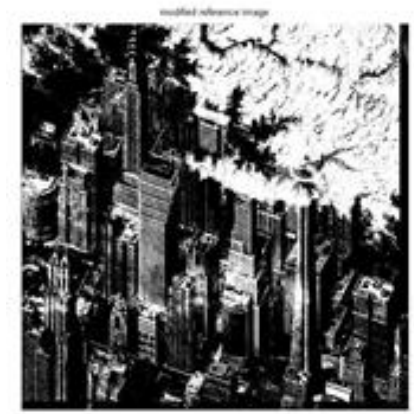

$f$

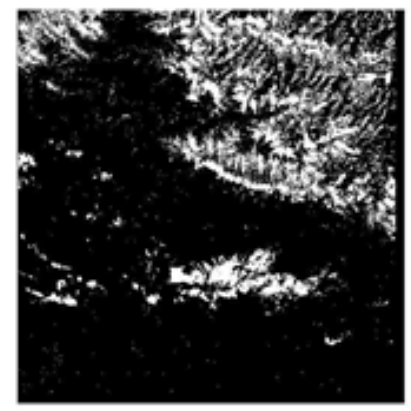

$g$

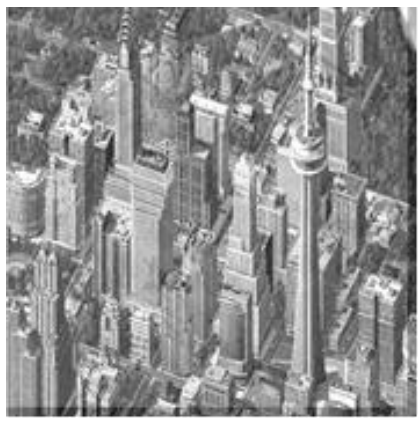

$h$

Fig: 5 (a) Original cloudy image; (b) Result of Multi scale Approach; (c) main image for no wavelet approach;(d) Reference Image ; (e)Modified Reference Image; (f) Detected Cloud Map without applying wavelet; (g) Cloud free Image for Fusion; (h) Fused Image with cloud Removal 


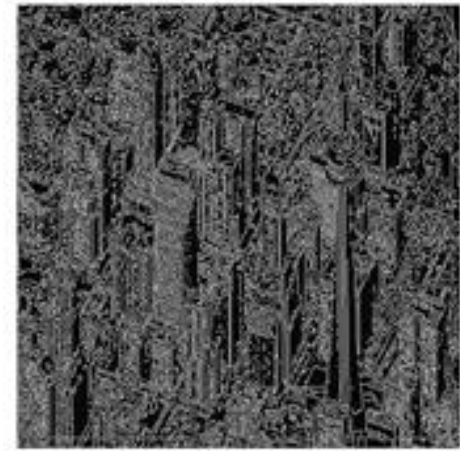

$i$

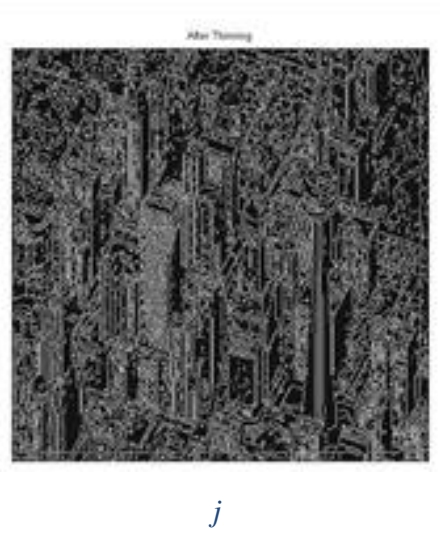

Fig: 5 (i) Extracted building edges with $1^{\text {st }}$ level wavelet transform; (j) Extracted building edges with $3^{\text {rd }}$ level wavelet transform

The respective results were shown in figure-5. As we can see in the results, cloud detection is achieved by the wavelet transform aided methods but in addition with cloud some other features of the respective image is also detected as clouds. The result obtained by multi-scale product approach is better than the simple undecimated wavelet transform approach of detection. The proposed approach is tested for the same set of SAR images and we obtained a better performance result as shown in the figure 5. This approach detects the clouds as well as the shadows the cloud from the image. After detection of cloud, following the procedure for image fusion as explained in prior section we obtained the result as shown in figure 5(i).

\section{CONCLUSION}

The algorithm used in this paper is a simple as well as effective technique of data fusion and edge detection by integrating complementary information of the source image. Wavelet analysis is a growing area in the field of remote sensing though it has very strong response in other areas of engineering.

\section{REFERENCES}

[1] Paul J. Gibson. Introductory Remote Sensing. Principles and Concepts. Rutledge, London, first edition, 2000.

[2] Christine Pohl. Geometric aspects of multisensor image fusion for topographic map updating in the humid tropics. PhD thesis, ITC, 1996.
[3] L.M.T. Carvalho, L.M.G. Fonseca, F. Murtagh, and J.G.P.W. Clevers. Digital change detection with the aid of multiresolution wavelet analysis. International Journal of Remote Sensing, 22(18):3871-3876, 2001.

[4] R. Todd Ogden. Essential wavelets for statistical applications and data analysis. Birkhauser, Boston, 1997.

[5] Rafael C. Gonzalez, Richard E. Woods, Steven L. Eddins, Digital Image Processing using MATLAB a book of Low Price Edition.

[6] Lucien Wald. Definitions and terms of reference in data fusion. International Archives of Photogrammetry and Remote Sensing, 32(Part 7-4-3 W6), June 1999.

[7] Christine Pohl. Tools and methods for fusion of images of different spatial resolution. International Archives of Photogrammetry and Remote Sensing, 32(Part 7-4-3 W6), June 1999.

[8] Paul M. Mather, University of Nottingham, Computer Processing of Remotely Sensed Images, third edition, John Willy \& Sons, Ltd.

[9] Bin Wang, Atsuo Ono, Kanako Maramatsu, and Noburo Fujiwara. Automated detection and removal of clouds and their shadows from landsat TM images. IEICE TRANS. INF.and SYST., E82-D(2), February 1999.

[10] V.K. Mehta, C.M. Hammock, and H. Krim. Data fusion of SSM/I channels using multiresolution wavelet transformation. North Carolina State University

[11] Oliver Rockinger. Pixel-level fusion of image sequences using wavelet frames. In Leeds University Press, editor, Proceedings of the 16 Leeds applied shape research workshop, Alt Moabit 96 A, 1996.Daimler Benz AG. Systems Technology Research, Intelligent Systems Group.

[12] Hong Wang, Zhongliang Jing, and Jianxun Li. Image fusion using non-separable wavelet frame. Chinese Optics Letters, 1(9), September 2003.

[13] Wu Xiuqing, Zhou Rong, Xu Yunxizng, “A Method of Wavelet-Based Edge Detection with Data Fusion for Multiple Images", Proceedings of the 3rd World Congress on Intelligent Control and Automation, July 2000 .

[14] Qi-Ming Qin, Si-Jin Chen, Wen-Jun Wang , De-Zhi Chen, Lin Wang, "The Building Recognition of High Resolution Satellite Remote Sensing Image Based On Wavelet Analysis", Proceedings of the Fourth International Conference on Machine Learning and Cybernetics, Guangzhou, 18-21 August 2005.

[15] Xiaoying Jin, Curt H. Davis, "Automated Building Extraction from High-Resolution Satellite Imagery in Urban Areas Using Structural, Contextual, and Spectral Information", EURASIPJournal on Applied Signal Processing 2005:14, 2196-2206.

[16] Sahoo T, Pattnaik S, "Cloud removal in Satellite Image using Auto Associative neural $\mathrm{n} / \mathrm{w}$ \& SWT . IEEE International Conference on Emerging Trends in Engineering and Technology, Nagpur, India July 16-18, 2008. 